\title{
LEFT VENTRICULAR PSEUDOANEURYSM - A COMPLICATION OF ACUTE MYOCARDIAL INFARCTION
}

\author{
Adnaldo Maia ${ }^{1}$, Rafael Tajra ${ }^{1}$, Adrian Rivadeneira ${ }^{1}$, Iuri António ${ }^{1}$, and Antônio de \\ Almeida ${ }^{1}$ \\ ${ }^{1}$ Dante Pazzanese Cardiological Institute
}

April 13, 2021

\begin{abstract}
Left ventricular pseudoaneurysm is a complication of acute myocardial infarction, especially after involvement of the inferior wall. The signs and symptoms are nonspecific. We report a case of a 69-year-old patient admitted for clinically important dyspnea. Transthoracic echocardiography showed pseudoaneurysm in the apical region, measuring 96x116 mm. Surgical correction was performed, using bovine pericardium and anchoring the ventricular orifice with 3-0 polypropylene sutures. It was not possible to perform coronary artery bypass grafting, as the distal beds of the proposed vessels were calcified. The patient was referred to the intensive care unit and discharged on the 17 th postoperative day.
\end{abstract}

\section{INTRODUCTION}

Ventricular pseudoaneurysm occurs when the complete cardiac rupture is contained by a strict layer of pericardium or fibrous tissue. Contrary to true aneurysms, it does not have myocardial or endocardial tissue. ${ }^{1}$ Among its etiologies, cardiac surgery, trauma, infectious endocarditis and myocardial infarction stand out, the latter being the main cause involved. ${ }^{2,3}$

It has a variable and insidious clinical presentation, developing in individuals with or without previous clinical events, especially in patients with acute myocardial infarction (AMI) due to involvement of the inferior wall. ${ }^{4,5}$ The surgical approach is an important therapy in view of the inherent complications. ${ }^{6}$

\section{CASE REPORT}

A 69-year-old male patient sought care in a cardiology emergency room for dyspnea, orthopnea and progressive edema of the lower limbs in the last months, with worsening of the condition. His pathological history revealed hypertension, ex-smoker (125 pack years), congestive heart failure and AMI about 1 month ago.

The patient was taking enalapril, bisoprolol, furosemide and rosuvastine. Upon physical examination on admission, he was in regular general condition, pale, anicteric and acyanotic. Physiological breath sounds with fine bilateral bursts audible in bases and middle third. Dyspneic, with $98 \% \mathrm{O}_{2}$ saturation in room air, not able to lie down. In the evaluation of cardiovascular system, regular heart sounds (S1, S2), without murmurs. Well-perfused ends.

Laboratory tests, electrocardiogram, transthoracic echocardiogram and cinecoronarioangiography (due to the history of AMI) were requested. The exams revealed hemoglobin $11.8 \mathrm{~g} / \mathrm{dl}$, hematocrit $37.0 \%$, platelets 306,000, INR (International Normalized Ratio) 1.20 and troponin 0.02. Admission electrocardiogram shows regular sinus rhythm with pathological $\mathrm{Q}$ waves in the inferior wall.

Transthoracic echocardiogram revealed significant left ventricular systolic dysfunction, with an ejection fraction of $31 \%$. In addition, an extensive pseudoaneurysm involving the apical region, measuring $96 \times 116 \mathrm{~mm}$ 
with neck measuring $35 \mathrm{~mm}$, associated with a diffuse moderate-sized pericardial effusion, with its largest layer measuring $16 \mathrm{~mm}$, adjacent to the right cardiac cavities, with incipient signs of hemodynamic repercussion.

As an urgent matter for coronary evaluation, it was decided to perform cinecoronarioangiography. A right coronary flow pattern was described, as well as a $40 \%$ calcified lesion in the proximal third of the right coronary. The anterior descendant exhibits marked $40 \%$ calcification of the proximal third with a $50 \%$ segmental lesion in the middle third. Circumflex artery had severe atheromatosis in its proximal third and occlusion in the middle third.

The patient was referred to the operating room, where the left common femoral artery was dissected and the right femoral vein was punctured. Heparinization and cannulation of these vessels were performed. In cardiopulmonary bypass, due to the risk of rupture, a hypothesis suggested by the presence of pericardial effusion on the preoperative echocardiogram, a median transsternal thoracotomy was performed, and the right great saphenous vein was dissected. In the intraoperative period, an important ventricular pseudoaneurysm was seen, however, there was no evidence of the pericardial effusion described on preoperative echocardiogram (Figure 1)

The pseudoaneurysm was approached through a circular incision bordering the normal wall. The cavity was disproportionate to the size of the left ventricle. Cerclage of the edge with fibrotic aspect was performed to reduce the orifice (Figure 2). The orifice, anchored in felt strips, was sutured with 16 stitches using polypropylene 3-0, followed by implantation of bovine pericardium with 3 layers. Finally, a linear suture was performed on the remaining tissue for better hemostatic control (Figure 3). 


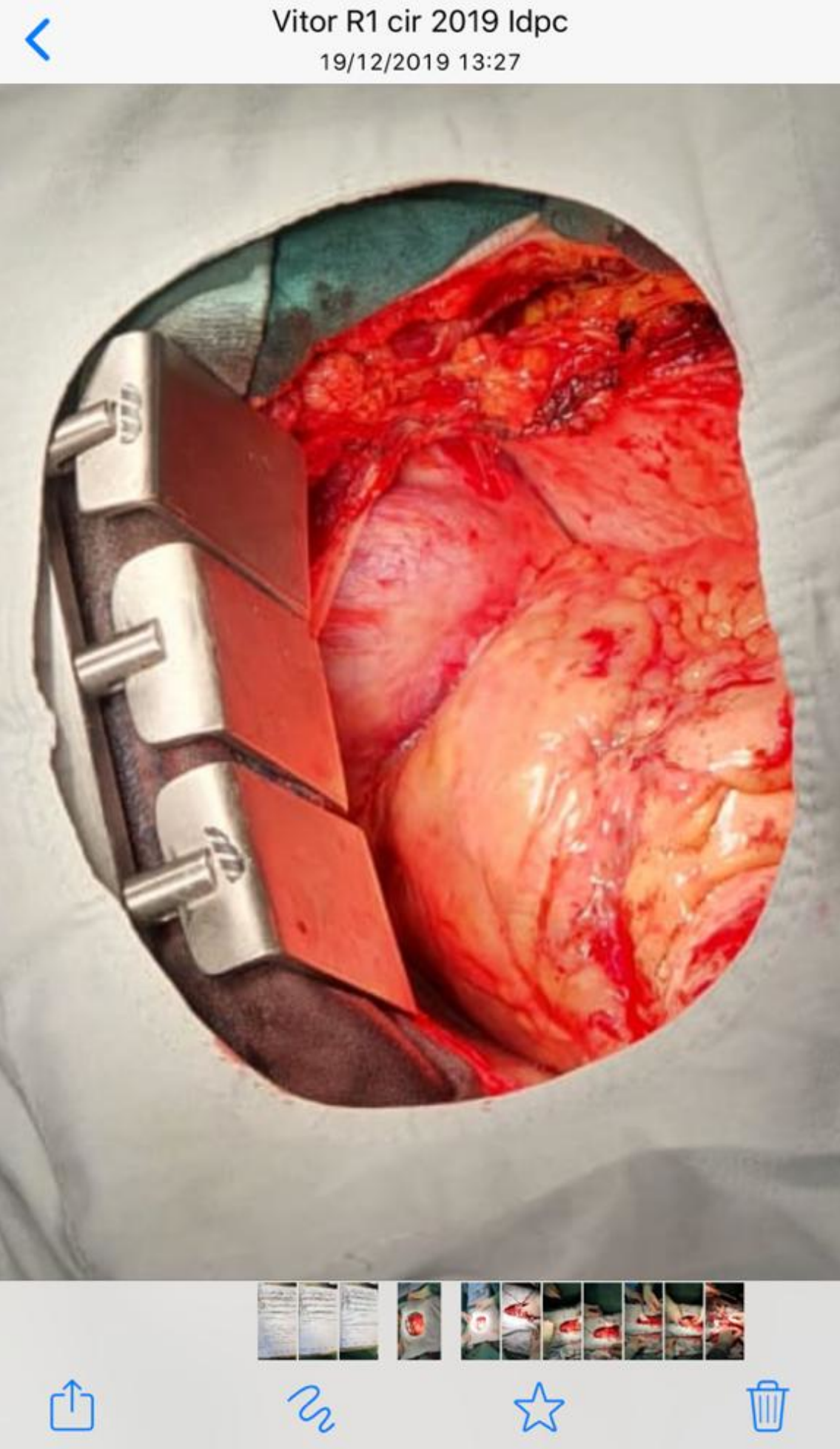

Figure 1: pseudoaneurysm after median transsternal thoracotomy 


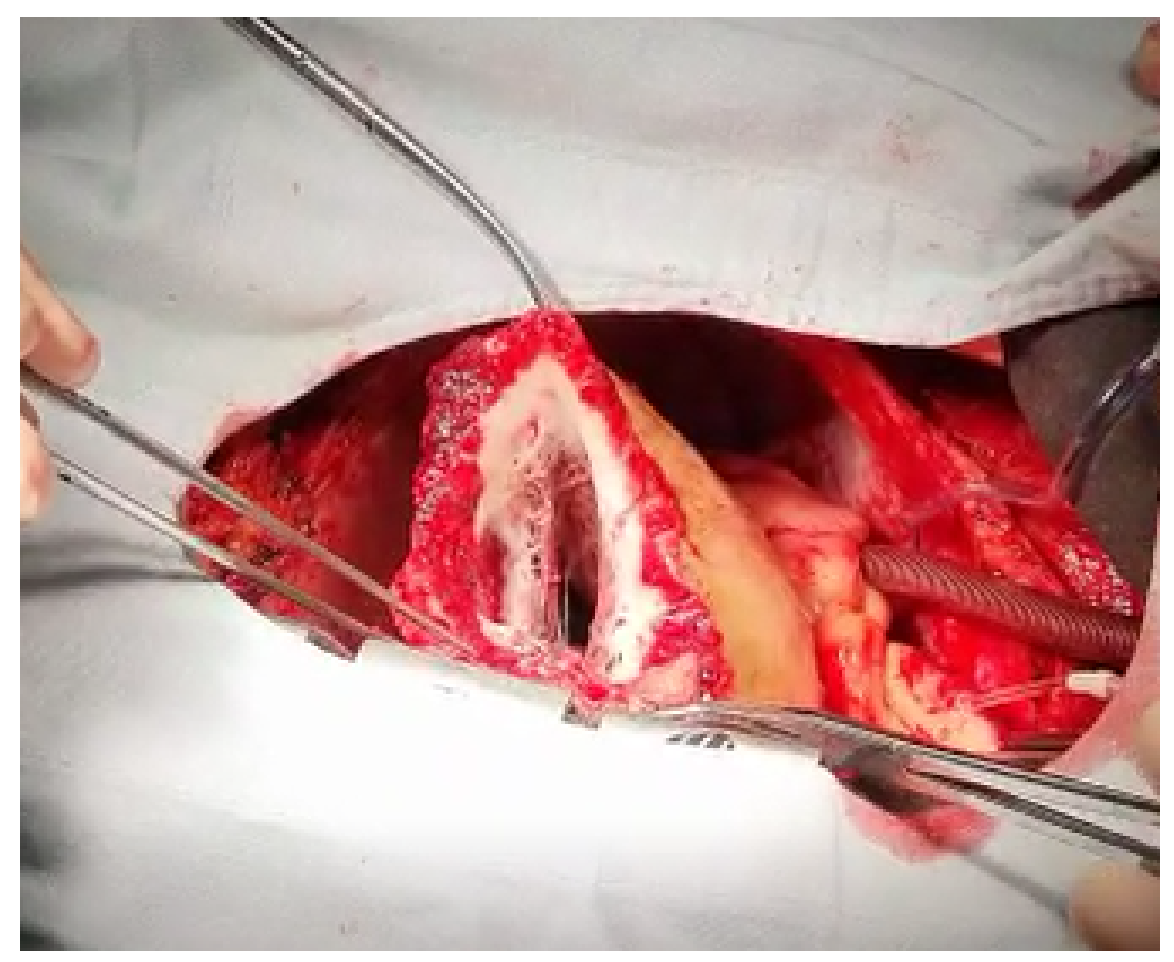

Figure 2 - Fibrotic aspect of the myocardial tissue in the topography of the ventricular pseudoaneurysm.

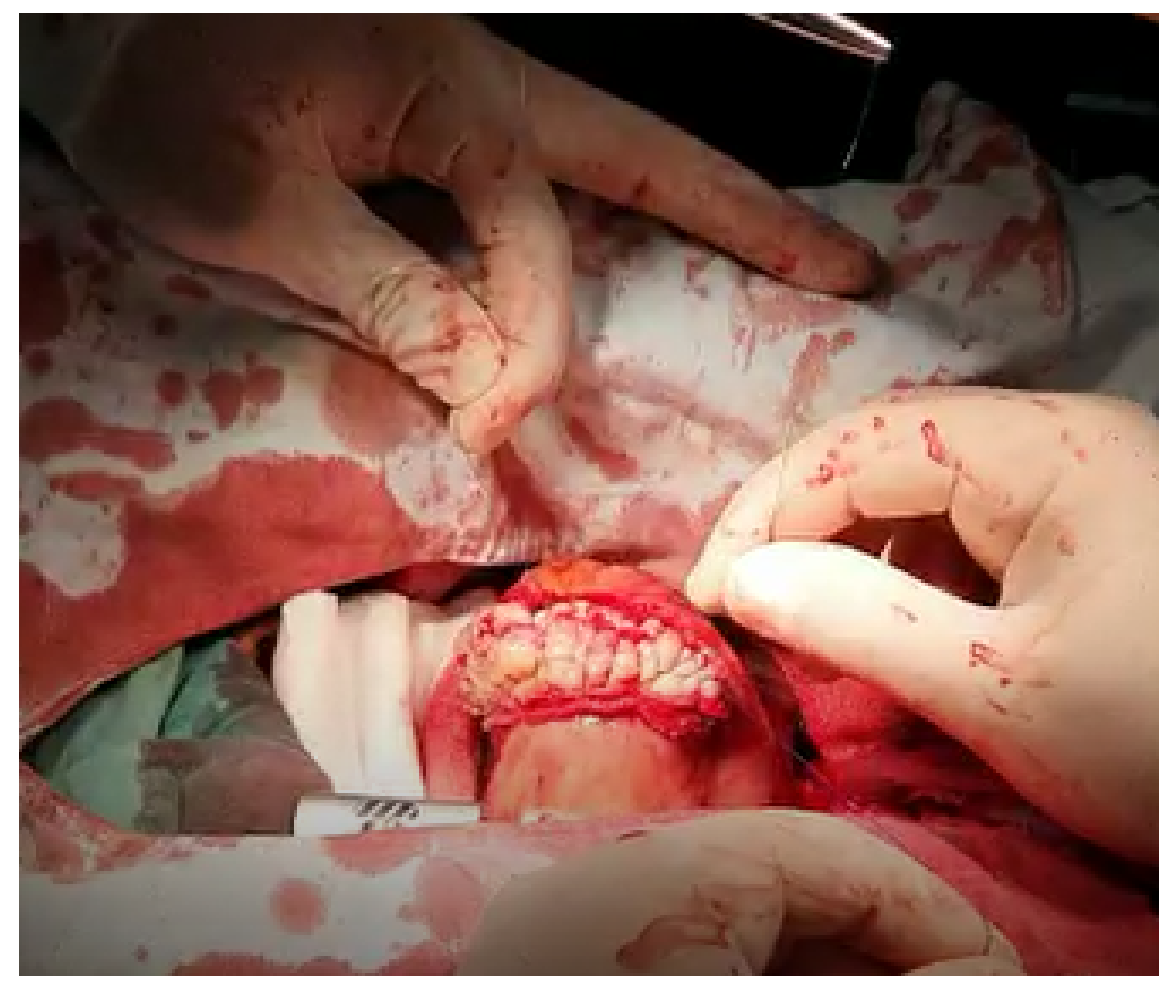

Figure 3 - Suture with bovine pericardium in 3 layers for correction of ventricular pseudoaneurysm. 
It was not possible to perform coronary artery bypass grafting, because the distal beds of the anterior descending artery were inadequate and there was an occlusion with significant calcification of the marginal artery.

After returning to normal heartbeat and adjusting blood volume, cardiopulmonary bypass was successfully discontinued. Cannula removal, protaminization, placement of mediastinal tube and pacemaker wire were performed next, in addition to hemostasis assessment and layered suture. The patient was then referred to the intensive care unit (ICU).

The patient evolved with progressive weaning of vasoactive drugs, with removal of mediastinal and right pleural tubes on the $4^{\text {th }}$ postoperative day. He was discharged on the $17^{\text {th }}$ postoperative day and remains stable under regular follow-up at the coronary clinic.

\section{DISCUSSION}

Left ventricular pseudoaneurysm occurs when the rupture of all cardiac layers is contained by pericardial adhesions or fibrotic tissue. Frances et al., ${ }^{1}$ in a systematic review in this group of patients, described that the main symptoms presented are congestive heart failure, chest pain and dyspnea, compatible with the signs verified in the present report.

Among the determining factors for the occurrence of pseudoaneurysm, AMI stands out, through rupture of the ventricular wall with absence of hemopericardium and cardiac tamponade, especially in AMI affecting the inferior wall ${ }^{2}$. The presentation can be late, varying from months to years. ${ }^{3}$

The risk of rupture demonstrated in cases of pseudoaneurysm producing hemorrhagic events is $35-40 \%$. However, due to the risk of imminent mortality, surgical approach is imperative after diagnosis. It is important to note that most cases are managed in the chronic phase and about $10 \%$ are asymptomatic. ${ }^{4}$

The surgical team opted for preoperative cinecoronarioangiography. Despite the diagnostic suspicion and the inherent risks, left ventriculography was not performed, which should be considered in the approach of these patients. ${ }^{5}$

\section{CONCLUSION}

The present report highlights the importance of considering pseudoaneurysm as a complication of myocardial infarction, referred to as a dilemma by some authors. ${ }^{6}$ The presentation and complementary exams are nonspecific and require suspicion by the assistant team. The resulting complications demand adequate surgical management to avoid them.

\section{AUTHOR CONTRIBUTIONS}

Adnaldo da Silveira Maia, MD - substantial contributions to research design, acquisition, analysis and interpretation of data; drafting the paper; approval of the submitted and final version.

Rafael Dib de Paulo Tajra, MD - substantial contributions to research design, acquisition, analysis and interpretation of data.

Iuri Betuel Gomes António, MD - substantial contributions to research design, acquisition, analysis and interpretation of data

Adrian Makarios Oviedo Rivadeneira, MD - substantial contributions to research design, acquisition, analysis and interpretation of data

Antônio Flávio Sanchez de Almeida, MD - approval of the submitted and final version.

\section{REFERENCES}

1. Frances, C; Romero, A; Grady, D. Left Ventricular Pseudoaneurysm. JACC Vol. 32, No. 3 September 1998:557-61. 
2. Moreno et al. Long term outcome of patients with postinfarction left ventricular pseudoaneurysm . Heart 2003;89:1144-1146.

3. Saidi et al. Left Ventricular Pseudoaneurysm Complicated With Very Late Rupture 5 Years After Myocardial Infarction . Jacc Case Reports, N4, 2019.

4. Faustino et al. Left ventricular pseudoaneurysm - a challenging diagnosis. Rev Port Cardiol. 2016;35(6):373.e1-37.

5. Ho, HH; Sinaga, DA; Lee, E; Watson, TJ; Hon, JK. Left ventricular pseudoaneurysm. Journal of Geriatric Cardiology (2017) 14: 78-80.

6. Bisoyi S, Dash AK, Nayak D, Sahoo S, Mohapatra R. Left ventricular pseudoaneurysm versus aneurysm a diagnosis dilemma . Ann Card Anaesth 2016;19:169-72

\section{ETHICS STATEMENT}

This manuscript followed the principles of the Declaration of Helsinki

and the Declaration of Istanbul. The appropriate informed consent

was obtained for the publication of this manuscript 